\title{
Design, Synthesis and Test of Novel Lipidated Cysteine Analogs as Efficient TLR2/6 Agonists
}

\author{
Yang Zhou, Abid H. Banday, Minying Cai, and Victor Hruby \\ Department of Chemistry and Biochemistry, University of Arizona, Tucson, AZ, 85721, USA
}

\section{Introduction}

Synthetic vaccines use synthetic molecules as antigens for vaccination. Since real pathogens are no longer needed in synthetic vaccines, they are considered safer than the traditional inactivated or attenuated vaccines. The development of synthetic vaccines can be achieved on the genome sequence of the pathogen, which is much more efficient than the traditional way of sampling pathogens from the outbreak and grow cultures. Despite these advantages, the development of synthetic vaccines is hindered by the poor immunogenicity of synthetic antigens and the lack of effective adjuvants that enhance immune responses [2].

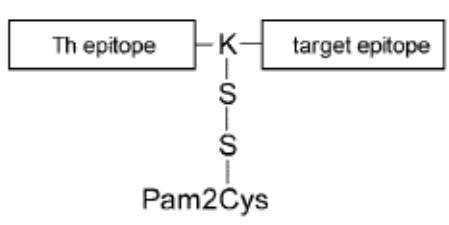

Fig. 1. Schematic

representation of Pam2Cys

based peptide vaccine [1].
$S$-[2,3-bis(palmitoyloxy)propyl]cysteine (Pam2Cys) was found to be a promising candidate as a synthetic vaccine adjuvant [1]. The molecule is found as the lipid component of macrophageactivating lipopeptide 2 (MALP-2) on the membrane of mycoplasma [3], and is able to activate the heterodimer TLR2/6 [1]. To make it a peptide vaccine adjuvant, Pam2Cys was conjugated to both a Th epitope and a target epitope through the $N$-terminal amino group and the $\varepsilon$-amino group of a lysine residue [1] (Figure 1). The construct was found to be able to induce both cytotoxic $\mathrm{T}$ cell immunity and antibody responses [1].

Nevertheless, the development of Pam2Cys based synthetic vaccine is hindered by the high cost of Pam2Cys synthesis. The synthesis involves orthogonal protection-deprotection techniques [4], which is labor-intensive. Here we report a novel synthetic pathway that can be used to effectively synthesize $N$-acetyl Pam2Cys analogues which share a similar structure as Pam2Cys (Figure 2). Our results suggest that $N$-acetyl Pam2Cys analogues are able to activate TLR2/6. Our in silico docking experiment shows that $N$-acetyl Pam2Cys analogues can interact well with the TLR2/6. $N$-acetyl Pam2Cys analogues are shown to be embedded deeper into the ligand binding pocket comparing to Pam2Cys. Our results suggest that $N$-acetyl Pam2Cys analogues, particularly $N$-acetyl Str2Cys, can serve as synthetic vaccine adjuvants with lower costs.
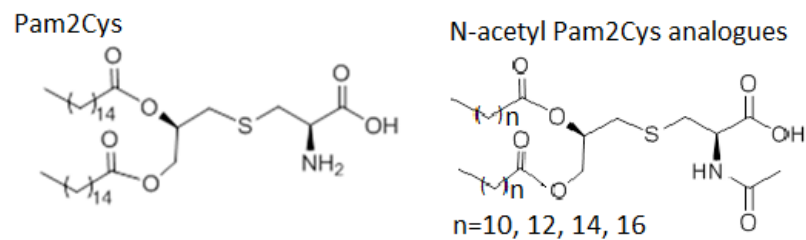

Fig. 2. Structures of Pam2Cys and N-acetyl Pam2Cys analogues.

\section{Results and Discussion}

To maximize the ability of $N$-acetyl Pam2Cys analogues to activate TLR2/6, molecules with varying numbers of carbons in the acyl groups $(18,16,14$ and 12) were synthesized and named as AHB-1, AHB-2, AHB-3 and AHB-4 accordingly. The TLR2/6 signaling was tested by an NF-kB based activation assay. Results show that AHB-1 ( $N$-acetyl $S$-[2,3-bis(stearyloxy)propyl]cysteine, $N$-acetyl Str2Cys) has the best ability to signal through TLR2/6 (Figure 3, Lane 1-4).

To further test if $N$-acetyl lipopeptides can trigger the TLR2 and TLR6 signaling as efficient as Pam2Cys, AHB-1 was conjugated with the short peptide SKKKK. The TLR2/6 signaling induced by $N$-acetyl Str2CSK4 (AHB-1SK4) and the commercially available compound Pam2CSK4 was measured. The results suggest that $N$-acetyl Str2CSK 4 achieves more than $60 \%$ of the TLR $2 / 6$ activation efficiency comparing to the Pam2CSK4 (Figure 3, Lane 5-6). 
To understand the effect of the extra $N$-acetyl group on lipopeptide binding to TLR2/6, in silico molecular docking experiments were performed. The structure of Pam2CysSK 4 bound to active TLR2/6 was previously determined [5] and was retrieved from Protein Data Bank (3A79). Since there are too many rotable bonds in Pam2CysSK4, which leads to inaccuracy in Glide flexible docking, the four lysine residues which according to the crystal structure do not interact with the receptor [5] were deleted and the diacyl chains were trimmed to six carbons to generate the initial conformation of Cap2CysSer ( $S$-[2,3-bis(capryloxy)propyl]cysteinyl-serine) molecule.

To reveal the conformation of $N$-acetyl Cap2CysSer when it binds to the TLR2/6 receptor, Glide docking with the docking flexible option was performed. This option allows all rotable bonds on the ligand to be able to rotate in order to find out the favorable conformations. Five conformations were found to be energetically favorable with the best docking score of -10.025 . Comparing to the initial position of Cap2CysSer, the $N$-acetyl Cap2CysSer is found to be more embedded into the ligand binding pocket of TLR2/6 (Figure 4). It suggests that the $N$-acetyl group on cysteine drives the lipopeptide to bury deeper into the binding pocket.

In conclusion, we developed new synthetic methods that can be used to synthesize $N$-acetyl Pam2Cys analogues. We showed that $N$-acetyl Str2Cys is able to activate TLR2/6, which makes it a promising candidate as a synthetic vaccine adjuvant. Our docking experiments provide information on the ligand-receptor interactions between $N$-acetyl Pam2Cys analogues and TLR2/6. Future work may still be required to establish the use of $N$-acetyl Str2Cys as a synthetic vaccine adjuvant.

\section{Acknowledgments}

This research was supported by U.S. Public Health Service, National Institutes of Health (GM104080).

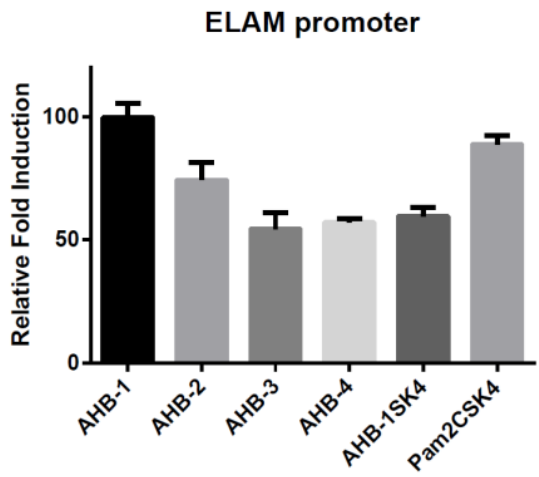

Fig. 3. TLR2/6 activation by $N$-acetyl Pam2Cys and analogues.

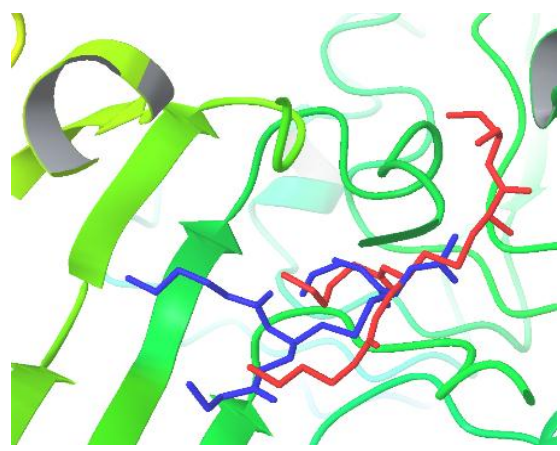

Fig. 4. Comparison of the best flexible docking result (lowest docking score) of $N$-acetyl Cap2CysSer (in blue) with the initial conformation of the Cap2CysSer (in red).

\section{References}

1. Jackson, D.C., Lau, Y.F., Le, T., Suhrbier, A., Deliyannis, G., Cheers, C., et al. Proc. Natl. Acad. Sci. USA 101, 15440-15445 (2004), http://dx.doi.org/10.1073/pnas.0406740101

2. Purcell, A.W., Zeng, W., Mifsud, N.A., Ely, L.K., Macdonald, W.A., Jackson, D.C. J. Pept. Sci. 9, 255-281 (2003), http://dx.doi.org/10.1002/psc.456

3. Mühlradt, P.F., Kiess, M., Meyer, H., Süssmuth, R., Jung, G. J. Exp. Med. 185, 1951-1958 (1997), http://dx.doi.org/10.1084/jem.185.11.1951

4. Zeng, W., Ghosh, S., Lau, Y.F., Brown, L.E., Jackson, D.C. J. Immunol. 169, 4905-4912 (2002), http://dx.doi.org/10.4049/jimmunol.169.9.4805

5. Kang, J.Y., Nan, X., Jin, M.S., Youn, S.J., Ryu, Y.H., Mah, S., et al. Immunity 31, 873-884 (2009), http://dx.doi.org/10.1016/i.immuni.2009.09.018 